\title{
Mingling of human and veterinary strains of Staphylococcus aureus: An emerging issue in health-care systems
}

Sara Giordana Rimoldi ${ }^{1 \neq}$, Annamaria Di Gregorio ${ }^{1 \neq}{ }$, Vittorio Sala ${ }^{2}$, Eleonora De Faveri ${ }^{2}$, Cristina Pagani ${ }^{1}$, Pietro Olivieri ${ }^{3}$, Claudio Savi ${ }^{4}$, Anna Lisa Ridolfo ${ }^{5}$, Antona Carlo ${ }^{6}$ and Maria Rita Gismondo ${ }^{7}$

1. Laboratory of Microbiology, Virology and Bioemergency, ASST Fatebenefratelli Sacco-Polo Universitario, Via G.B.

Grassi 74, 20157 Milan, Italy; 2. Department of Veterinary Sciences and Public Health, Università di Milano, Italy;

3. Medical Management Staff, ASST Fatebenefratelli Sacco-Polo Universitario, Via G.B. Grassi 74, 20157 Milan, Italy;

4. Cardiac Surgical Intensive Care Unit, ASST Fatebenefratelli Sacco-Polo Universitario, Via G.B. Grassi 74, 20157 Milan, Italy; 5. Department of Infectious Diseases, ASST Fatebenefratelli Sacco-Polo Universitario, Via G.B. Grassi 74, 20157 Milan, Italy; 6. Cardiac Surgery Unit, ASST Fatebenefratelli Sacco-Polo Universitario, Via G.B. Grassi 74, 20157 Milan, Italy; 7. Laboratory of Microbiology, Virology and Bioemergency, ASST Fatebenefratelli Sacco-Polo Universitario, Via G.B. Grassi 74, 20157 Milan, Italy.

Corresponding author: Sara Giordana Rimoldi, e-mail: sara.rimoldi@asst-fbf-sacco.it Co-authors: AD: annadigre@yahoo.it, VS: vittorio.sala@unimi.it, ED: defaverieleonora@gmail.com, CP: cristina.pagani@asst-fbf-sacco.it, PO: pietro.olivieri@asst-fbf-sacco.it, CS: claudio.savi@asst-fbf-sacco.it, ALR: annalisa.ridolfo@asst-fbf-sacco.it; AC: carlo.antona@unimi.it, MRG: mariarita.gismondo@unimi.it ${ }^{\ddagger}$ Rimoldi and Di Gregorio equally contributed to the work.

Received: 03-08-2017, Accepted: 30-10-2017, Published online: 28-11-2017

doi: 10.14202/IJOH.2017.77-82 How to cite this article: Rimoldi SG, Di Gregorio A, Sala V, De Faveri E, Pagani C, Olivieri P, Savi C, Ridolfo AL, Carlo A, Gismondo MR. Mingling of human and veterinary strains of Staphylococcus aureus: An emerging issue in health-care systems. Int J One Health 2017;3:77-82.

\begin{abstract}
Aim: Methicillin-resistant Staphylococcus aureus remains a leading cause of hospital and community infections. We report a retrospective molecular characterization of $S$. aureus strains from different settings: hospital workers and patients, and veterinarian surgeons and pets.
\end{abstract}

Materials and Methods: Eighty-nine $S$. aureus isolates obtained from nasal swabs of 10 patients, 17 health-care workers (HCWs), 9 pets, and 53 veterinarians were genotypically characterized by means of repetitive extragenic palindromic polymerase chain reaction (Rep PCR) and whole-genome sequencing.

Results: Thirteen different sequence types (STs) were detected: ST398, ST22, ST8, ST30, ST15, ST5, ST121, ST45, ST10, ST6, ST34, ST97, and ST1. Two new STs differing from ST22 and ST5 for a single multilocus sequence typing gene were also identified. Rep PCR documented a genetic relationship among isolates obtained from 5 veterinarians and $10 \mathrm{HCWs.}$

Conclusion: The large diversity of $S$. aureus strains detected may reflect a larger epidemiology within the hospital and community, in which companion animals likely act as a reservoir. We identified the circulation of ST5, ST8, ST15, ST22, ST30, ST45, and ST121 both in the hospital and veterinarian environment. Starting from the idea of a unique setting where our population lives, we consider the relationship between community- and hospital-acquired S. aureus.

Keywords: health-care workers, multilocus sequence typing, S. aureus, single-nucleotide polymorphisms, pets, veterinarians.

\section{Introduction}

Staphylococcus aureus is a major cause of healthcare (HA)-associated infections worldwide [1,2]. It is one of the most prevalent causes of nosocomial bacteremia, hospital-acquired pneumonia, and surgical site infections, mainly in the intensive care settings $[1,2]$. In addition, methicillin-resistant $S$. aureus (MRSA), firstly reported in 1961, has quickly become one of the most important antibiotic-resistant nosocomial pathogens globally $[3,4]$. In one-third of the European countries, including Italy, MRSA continues to account for more than $25 \%$ of bloodstream infections despite

Copyright: Rimoldi, et al. This article is an open access article distributed under the terms of the Creative Commons Attribution 4.0 International License (http://creativecommons.org/licenses/ by/4.0/), which permits unrestricted use, distribution, and reproduction in any medium, provided you give appropriate credit to the original author(s) and the source, provide a link to the Creative Commons license, and indicate if changes were made. The Creative Commons Public Domain Dedication waiver (http:// creativecommons.org/ publicdomain/zero/1.0/) applies to the data made available in this article, unless otherwise stated. efforts in infection control [5]. Nasal and extranasal carriage of MRSA often leads to invasive MRSA infection, as MRSA colonization is associated with an increased risk of acquiring MRSA infection during hospital stays [6]. Moreover, the pressure of colonization in health-care environment plays an important role in the subsequent dissemination of MRSA strains [7].

Although MRSA has long been considered an issue confined to health-care environment (HA-MRSA), its epidemiology has become more complex in the recent years. In the early 90s, MRSA strains genetically distinct from HA-MRSA have been recovered increasingly in the community from patients without previous HA contact (community-acquired [CA] - MRSA) [8,9]. Notably, CA-MRSA frequently carries genes for the cytotoxin Panton-Valentine leukocidin (PVL) that confers enhanced virulence [8,9].

MRSA which originated in the community and is associated with exposure to livestock ([LA]MRSA) has also emerged in many countries $[10,11]$. 
LA-MRSA has shown the capacity to colonize humans and cause zoonotic infections in farm workers, abattoir workers, and veterinarians [10].

Furthermore, MRSA is increasingly reported in veterinary medicine, particularly, in small animals and equine practices [12-14]. In general, companion animal strains of MRSA differ from those in livestock and meat production animals. This is probably because in companion animals, MRSA infection is primarily a human disease, with the strains carried by human owners being passed to their animals [15]. Historically, MRSA infections in companion animals involved strains resembling human nosocomial strains, including epidemic MRSA [12]. When these epidemic HA-MRSA clones were observed in dogs [12-14], the assumption was that the direction of spread had been from humans to animals. However, this situation is changing rapidly, with strains of MRSA that is thought to have evolved in animals colonizing and infecting human attendants [16]. Like their companion animals, companion humans are more often colonized than infected, providing a reservoir for reinfection of their human and animal companions [17-19]. Human skin scales with MRSA are easily shed from leg ulcers, eczematous skin, and pressure areas during activities of daily living. Undetected colonized animals can provide a reservoir for continuing relapsing infection in humans [18-21].

Overall, therefore, MRSA can no longer be regarded as an exclusively HA problem, and it cannot be fought by hospital infection prevention and control measures alone. The expanding community reservoir of MRSA leads, in fact, to the inevitable infiltration and diffusion of CA-MRSA into hospitals through colonized/infected patients, visitors, and health-care workers (HCWs) [21-25]. Consequently, identifying reservoirs and tracking the source of MRSA diffusion within hospitals have become more difficult. Limitation in pathogens typing techniques available in routine clinical practice further hinders such investigations.

Molecular characterization methods are an essential tool for discriminating between isolates of epidemiologically important microorganisms. A variety of molecular typing methods [26,27] can be independently used to classify MRSA strains, including pulsed-field gel electrophoresis, multilocus sequence typing (MLST), or Staphylococcus protein A (spa) typing by sequencing the highly polymorphic spa gene. Spa type possesses high throughput and good interlaboratory reproducibility but can characterize $S$. aureus isolates within limited discrimination of a clonal complex. Greater discrimination such as provided with whole-genome sequencing (WGS) and single-nucleotide polymorphism (SNP) analysis could be useful to discern outbreak from non-outbreak strains in settings where similar strains are collected within a short timeframe [28,29].
This study was designed to investigate retrospectively whether $S$. aureus circulation in the cardiac-surgery area of ASST Fatebenefratelli-Sacco Hospital in Milan results from circulation/transmission of a heterogeneous mixing of $S$. aureus strains including those potentially originated from community/animal reservoirs. The causing of this rating was the occurrence during April 2015 of four cases of deep sternal wound infections (DSWIs) caused by S. aureus. The discovery of similar strains in some HCWs and veterinarians enrolled for a previous study [30] led us to extend our investigation on MRSA clones circulating among veterinarians and companion animals assisted by them.

\section{Materials and Methods}

\section{Ethical approval}

All examined isolates were cultured and preserved as part of the routine diagnostics (standard care) and local epidemiological surveillance regulations. Written informed consent for routine diagnostic and medical/epidemiological procedures was collected for each patient and healthcare operator. All data used in the study were previously anonymized, according to the requirements set by Italian Data Protection Code (leg. Decree 196/2003) and by the general authorizations issued by the Data Protection Authority. Approval by the Ethics Committee was therefore not required.

\section{Study population}

From January to December 2015, 365 nasal swabs collected from HCWs (98 physicians, nurses, and operating room and Intensive Care Unit (ICU) personnel), patients (10 subjects with DSWI), veterinarians (192), and pets (52 dogs and 13 cats) were investigated for the presence of $S$. aureus by the Laboratory of the Microbiology, Virology, and Bioemergency ASST Fatebenefratelli-Sacco Hospital, Milan, Italy. S. aureus isolates were characterized by means of antimicrobial susceptibility testing and genotyping techniques.

\section{Microbiology}

Nasal swabs were plated on Mannitol salt plates (BioMérieux, Marcy l'Etoile, France) that were incubated at $37^{\circ} \mathrm{C}$ overnight. Identification at the species level and antimicrobial susceptibility was determined using the Vitek2 automated system (BioMérieux) considering the criteria of the European Committee on Antimicrobial Susceptibility Testing breakpoints [31]. Isolates were stored at $-80^{\circ} \mathrm{C}$.

\section{Real-time polymerase chain reaction (PCR)}

Cultures were incubated on Columbia blood agar (BioMérieux). S. aureus DNA was extracted with the automatic extractor Easymag (BioMérieux) and analyzed with a real-time PCR (RealCycler SAMAPV, Progenie Molecular, Valencia, Spain) to detect the presence of the mecA gene, coding for methicillin resistance, and the PVL toxin gene. 


\section{Repetitive extragenic palindromic PCR (Rep-PCR)}

Seventy-eight $S$. aureus isolates (7 methicillin-sensitive $S$. aureus [MSSA] and 71 MRSA) of HCWs, veterinaries, and pets were genotyped using an automated Rep-PCR (DiversiLab system; BioMérieux). Band patterns were compared by means of Pearson's correlation and modified KullbackLeibler methods using the DiversiLab web-based software. The isolates whose band patterns were at least 95\% similar were considered to be genetically related.

\section{Next-generation sequencing (NGS)}

Eighty-nine $S$. aureus isolates (9 MSSA and 80 MRSA) collected from HCWs, patients, veterinarians, and pets were fully sequenced with NGS on the Illumina MiSeq platform (kit v3, 600 cycles). Each of these isolates underwent molecular typing by an MLST, and 27 (17 HCWs and 10 patients who shared the same sequence types [STs]) of them underwent SNP analysis. WGS was performed at the Histology and Molecular Biology Section of the Army Medical Research Center in Rome.

MLST compared single nucleotide variants within $7 \mathrm{~S}$. aureus reference genes (arcC, aroE, glpF, gmk, pta, tpiA, and yqiL) to a reference database [32]. WGS was used to obtain at the same time in silico data related to MLST and SNP analysis. We conducted in silico MLST analysis using the resources from the Center for Genomic Epidemiology (http://www. genomicepidemiology.org).

Once all alleles were assigned a designation for the 7 MLST genes, an ST pattern was defined, comparing the whole-genome sequences against the MLST database.

SNP analysis was performed using the program kSNP v2.1.2 that defines SNP loci by an oligo of length $\mathrm{k}$, identified by the Kchooser script, surrounding a central SNP allele [33]. A core SNP matrix including only SNPs detected at the loci that were present in all genomes was determined, and a maximum likelihood tree was visualized with the software Dendroscope v3.2.10 [34].

\section{Results}

\section{Patient and HCW evaluation}

From January to December 2015, 10 patients hospitalized in the cardiac-surgery area of our hospital had $S$. aureus isolated from surgical wound, including a cluster of 4 cases observed during April 2015. All the 10 patients were positive for $S$. aureus from nasal swabs. Two of $10 \mathrm{~S}$. aureus strains were MSSA (mecA negative), whereas 8 were MRSA (mec $A$ positive).

As part of an epidemiological investigation, 98 HCWs operating in the cardiac-surgery and ICU underwent nasal screening for $S$. aureus, and $17(12 \%)$ resulted positive: 7 (41\%) for MSSA and $10(59 \%)$ for MRSA. All $S$. aureus isolates from patients and HCWs were negative for PVL gene.

WGS was performed to assess relationships among strains isolated from patients and HCWs.
In silico extrapolation of MLST profiles of 27 whole genomes revealed 10 different STs: the most frequent was ST22 (12 strains), followed by ST121, ST30, ST8, and ST5 (2 strains each) and ST6, ST10, ST15, ST34, and ST45 (1 strain each). Two new STs differing from ST22 and ST5 for a single MLST gene were also identified (Table-1). In addition, SNP analysis allowed us to discriminate between samples with the same ST, such as in the case of the most predominant ST22 group (Figure-1).

WGS discounted patient-to-patient transmission as the only origin of the cluster of DWSI which occurred during April 2015. In fact, only 2 of 4 patients had the same ST (ST22) and similarities at SNP analysis, whereas the other 2 belonged to ST5 or ST30 clones. However, similarities of ST strains were detected among patients and HCWs: 3 patients and 3 HCWs shared the same ST22 strain, and 2 patient and HCWs pairs shared the same ST8 and ST30 strains, respectively.

\section{Veterinarian and pet evaluation}

Overall, 53 of $192(27.6 \%)$ nasal swabs from veterinarians and 9 of $53(16.9 \%)$ samples from pets were positive for MRSA. Among 62 whole genomes, in silico extrapolation of MLST profiles revealed 9 different STs: The most frequent was ST398 (45 strains),

Table-1: S. aureus (MSSA or MRSA) strains and MLST profiles detected in patients and operators in the cardiac-surgical area of L. Sacco Hospital, Milan, from January to December 2015.

\begin{tabular}{|c|c|c|c|}
\hline $\begin{array}{l}\text { ID } \\
\text { analysis }\end{array}$ & $\begin{array}{l}\text { Patient/operator } \\
\text { ID }\end{array}$ & $\begin{array}{l}\text { S. aureus } \\
\text { strain }\end{array}$ & MLST \\
\hline MRSA1 & Operator 1 & MRSA & ST22 \\
\hline MRSA2 & Patient 1 & MRSA & ST22 \\
\hline MSSA3 & Patient 2 & MSSA & ST22 \\
\hline MRSA4 & Operator 2 & MSSA & ST121 \\
\hline MSSA5 & Patient 3 & MSSA & New allele ST5 \\
\hline MRSA6 & Operator 3 & MRSA & ST121 \\
\hline MRSA7 & Operator 4 & MRSA & ST22 \\
\hline MRSA8 & Operator 5 & MRSA & ST22 \\
\hline MRSA9 & Operator 6 & MRSA & ST22 \\
\hline MRSA10 & Patient 4 & MRSA & ST5 \\
\hline MRSA11 & Operator 7 & MSSA & New allele ST22 \\
\hline MRSA12 & Operator 8 & MSSA & ST10 \\
\hline MRSA13 & Operator 9 & MSSA & ST15 \\
\hline MRSA14 & Operator 10 & MSSA & ST8 \\
\hline MRSA15 & Operator 11 & MSSA & ST30 \\
\hline MRSA16 & Operator 12 & MSSA & ST45 \\
\hline MRSA17 & Patient 5 & MRSA & ST8 \\
\hline MRSA18 & Patient 6 & MRSA & ST22 \\
\hline MRSA19 & Patient 7 & MRSA & ST30 \\
\hline MRSA20 & Patient 8 & MRSA & ST22 \\
\hline MRSA21 & Patient 9 & MRSA & ST22 \\
\hline MRSA22 & Patient10 & MRSA & ST5 \\
\hline MRSA23 & Operator 13 & MRSA & ST22 \\
\hline MRSA24 & Operator 14 & MRSA & ST22 \\
\hline MRSA25 & Operator 15 & MRSA & ST34 \\
\hline MRSA26 & Operator 16 & MRSA & ST6 \\
\hline MRSA27 & Operator 17 & MRSA & ST22 \\
\hline
\end{tabular}

MSSA = Methicillin-sensitive Staphylococcus aureus, MRSA = Methicillin-resistant Staphylococcus aureus, S. aureus =Staphylococcus aureus, MLST: Multilocus sequence typing 


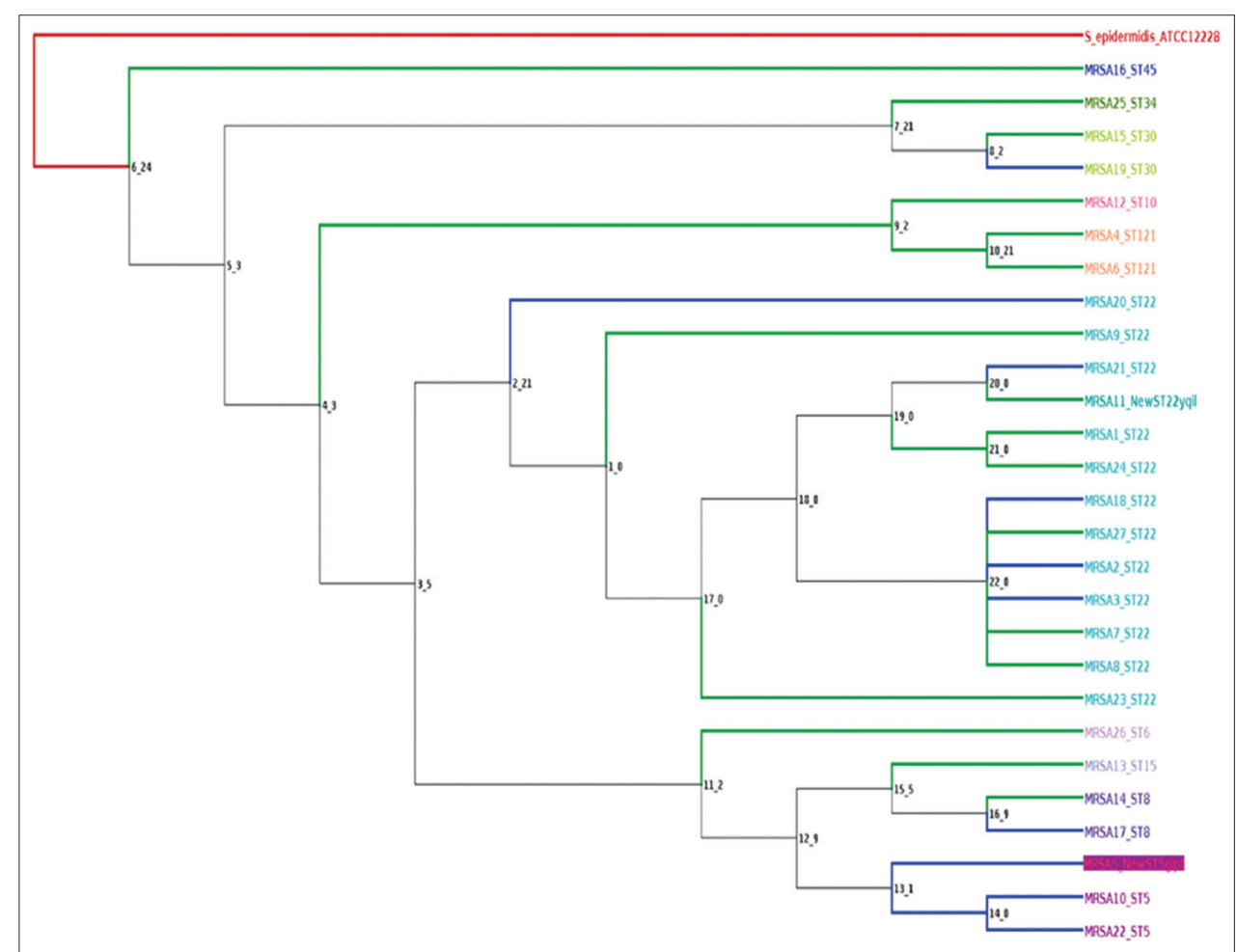

Figure-1: Dendrogram resulting from single-nucleotide polymorphisms analysis of Staphylococcus aureus strains.

followed by ST8, ST15, ST 45, and ST22 (2 strains each) and ST1, ST5, ST10, ST30, and ST97 (one strain each).

Even though 2 different predominant STs were found in the 2 settings of our study (ST398 for veterinarians and ST22 for HCWs and patients), the discovery of strains typical of animals (ST121 and ST45) in HCWs and the finding of some STs both in HCWs and veterinarians (ST5, ST8, ST10, ST15, ST22, and ST30) led us to investigate the clonal relationship between our hospital strains and those present in the veterinarian setting. It is interestingly to note that two HCWs carrying strains typical of animals lived with companion animals (a cat and a dog, respectively), but nasal swabs taken from their pets resulted negative.

\section{Rep PCR evaluation}

The clonal relationship among the 27 S. aureus strains obtained from HCWs and patients and the 62 obtained from veterinarians and pets was investigated by means of Rep-PCR. Using the Diversilab analysis software, we were able to obtain a hierarchical cluster representation of genomic similarities between this large number of samples. Overall, isolates presented a low similarity of sequence, confirming the results of MLST analysis.

With regard to the STs shared by veterinarians and HCWs, there was a correlation between human and animal ST121, and high similarity was found for ST45 shared by one HCW and one cat. Moreover, we observed the presence of indistinguishable strains (belonging to the same pattern) and related strains (belonging to different patterns, but assimilated to the same group) among HCWs and veterinarians.
A sharing pattern was reported in 3 situations: 1 case among 5 HCWs (ST22) and 1 veterinarian (ST unknown) and 2 cases among $1 \mathrm{HCW}$ and 1 veterinarian (HCW ST10 and veterinary ST10). In the other 2 situations, the strains belonged to different patterns but were related in the same group: 1 case among 2 HCWs and 1 veterinarian and another among $1 \mathrm{HCW}$ (ST15) and 1 veterinary (ST15).

\section{Discussion}

Increasing human and animal reservoirs outside the HA environment make more difficult to understand and control the diffusion of MRSA in hospitals. CA-MRSA is replacing classic hospital MRSA clones in many countries and have higher potential in transmission and virulence than HA-MRSA clones $[8,9]$. This poses new challenges for infection control, and physicians need to be aware of and understand the burden of this problem.

Molecular typing is essential for epidemiological studies and outbreak investigations. To this end, WGS has high discriminatory power and resolution and is well placed to become the gold standard in bacterial typing [27]. We used WGS to characterize bacterial isolates, which facilitated our understanding of the circulation of $S$. aureus in the cardiac-surgery area of our hospital and documented that patient-to-patient transmission was not the only pattern of diffusion. However, we were not able to establish whether transmission had taken place between HCWs and patients or vice versa.

WGS revealed ST121 and ST45 in two HCWs who lived with pets (cat and dog), but nasal swabs 
from their animals resulted negative. However, the genomic identity between isolates obtained from dogs and cats and their owners has previously been documented in the literature, supporting the role of pets as reservoirs of CA-MRSA strains and their possible contribution to the maintenance and mixing of locally circulating strains [17-21].

Further investigation led us to seek clonality among the strains detected in our hospital and those found in veterinarians and pets. Rep-PCR revealed a correspondence between a cat and 1 of the $2 \mathrm{HCWs}$ who lived with a cat. A high similarity (the same pattern or the same group) was also found among isolates from 5 veterinarians and $10 \mathrm{HCWs}$.

All these findings could reinforce the concept of an evolving epidemiology of $S$. aureus which involves both humans and animals [35]. The new epidemiology of MRSA, in particular, is closely related to the different environments of the animal species and humans with possible exchange of strains among the different epidemiological settings (community, hospital, and livestock).

\section{Conclusion}

Starting from the epidemiological circulation of infection in humans and animals and a unique setting where our population lived in close proximity (hospital and veterinarian workers, patients and pets), we considered the relationship between CA- and HA$S$. aureus. In this context, the most effective strategy was the monitoring of circulating strains to track and locate them. For this reason, further investigations are needed to genomically characterize better the strains identified, especially the novel ones. For better epidemiological evaluation, it would be appropriate to expand the number of patients enrolled in the study. HA-MRSA, CA-MRSA, and LA-MRSA are an important novel problem that needs to be considered.

\section{Authors' Contributions}

SGR and AMD: Conception and design, laboratory work, data analysis, and manuscript write up. VS, ALR, and ED: Conception, design, and review of the manuscript. CA and MRG: Critical review of the manuscript. CS and PO: Patient's enrolment. All authors read and approved the final manuscript.

\section{Acknowledgments}

The authors would like to thank Dr. Bernardina Gentile and Dr. Florigio Lista for their contribution to the molecular biology (sequencing). The authors are thankful to Histology and Molecular Biology Section, Army Medical Research Center, Via Santo Stefano Rotondo 4, 00184 Rome, Italy for providing necessary facilities for this study.

\section{Competing Interests}

The authors declare that they have no competing interests.

\section{References}

1. Weiner LM, Webb AK, Limbago B, Dudeck MA, Patel J, Kallen AJ, et al. Antimicrobial-resistant pathogens associated with healthcare-associated infections: Summary of data reported to the national healthcare safety network at the centers for disease control and prevention, 2011-2014. Infect Control Hosp Epidemiol 2016;37:1288-301.

2. Vincent JL, Rello J, Marshall J, Silva E, Anzueto A, Martin $\mathrm{CD}$, et al. International study of the prevalence and outcomes of infection in intensive care units. JAMA 2009;302:2323-9.

3. Chambers HF, Deleo FR. Waves of resistance: Staphylococcus aureus in the antibiotic era. Nat Rev Microbiol 2009;7:629-41.

4. DeLeo FR, Chambers HF. Reemergence of antibiotic-resistant Staphylococcus aureus in the genomics era. J Clin Invest 2009;119:2464-74.

5. de Kraker ME, Jarlier V, Monen JC, Heuer OE, van de Sande N, Grundmann H. The changing epidemiology of bacteraemias in Europe: Trends from the European antimicrobial resistance surveillance system. Clin Microbiol Infect 2013;19:860-8.

6. Kluytmans J, van Belkum A, Verbrugh H. Nasal carriage of Staphylococcus aureus: Epidemiology, underlying mechanisms, and associated risks. Clin Microbiol Rev 1997; 10:505-20.

7. Williams VR, Callery S, Vearncombe M, Simor AE. The role of colonization pressure in nosocomial transmission of methicillin-resistant Staphylococcus aureus. Am J Infect Control 2009;37:106-10.

8. DeLeo FR, Otto M, Kreiswirth BN, Chambers HF. Community-associated methicillin-resistant Staphylococcus aureus. Lancet 2010;375:1557-68.

9. David MZ, Daum RS. Community-associated methicillin-resistant Staphylococcus aureus: Epidemiology and clinical consequences of an emerging epidemic. Clin Microbiol Rev 2010;23:616-87.

10. van Cleef BA, Monnet DL, Voss A, Krziwanek K, Allerberger F, Struelens M, et al. Livestock-associated methicillin-resistant Staphylococcus aureus in humans, Europe. Emerg Infect Dis 2011;17:502-5.

11. Chuang YY, Huang YC. Livestock-associated meticillin-resistant Staphylococcus aureus in Asia: An emerging issue? Int J Antimicrob Agents 2015;45:334-40.

12. Rich M, Roberts L, Kearns AM. Methicillin-resistant Staphylococcus aureus isolates from companion animals. Vet Microbiol 2005;105:13-4.

13. Loeffler A, Boag AK, Sung J, Lindsay JA, Guardabassi L, Dalsgaard A, et al. Prevalence of methicillin-resistant Staphylococcus aureus among staff and pets in a small animal referral hospital in the UK. Antimicrob Chemother 2005; 109:285-96.

14. Morris D, Rook KS, Shofer FS, Rankin SC. Screening of Staphylococcus aureus, Staphylococcus intermedius and Staphylococcus schlieferi isolates obtained from small companion animals for antimicrobial resistance: A retrospective review of 749 isolates (2003-2004). Vet Dermatol 2006;5:332.

15. Morgan M. Methicillin-resistant Staphylococcus aureus and animals: Zoonosis or humanosis? J Antimicrob Chemother 2008;62:1181-7.

16. Van Belkum A, Melles DC, Peeters JK, van Leeuwen WB, van Duijkeren E, Huijsdens XW, et al. Methicillin-resistant Staphylococcus aureus sequence type 398 in pigs and humans. Emer Infect Dis 2008;14:479-83.

17. Cefai C, Ashurst S, Owens C. Human carriage of methicillin-resistant Staphylococcus aureus linked with pet dog. Lancet 1994;344:539-40.

18. Manian FA. Asymptomatic nasal carriage of mupirocin resistant methicillin-resistant Staphylococcus aureus (MRSA) in a pet dog associated with MRSA infection in 
household contacts. Clin Infect Dis 2003;36:e26-8.

19. van Duijkeren E, Wolfhagen MJ, Heck ME, Wannet WJ. Transmission of a Panton-Valentine leucocidin-positive, methicillin-resistant Staphylococcus aureus strain between humans and a dog. J Clin Microbiol 2005;43:6209-11.

20. Sing A, Tuschak C, Hörmansdorfer S. Methicillin-resistant Staphylococcus aureus in a family and its pet cat. N Engl J Med 2008;358:1200-1.

21. Scott GM, Thomson R, Malone-Lee J, Ridgway GL. Crossinfection between animals and man: Possible feline transmission of Staphylococcus aureus infection in humans? J Hosp Infect 1988;12:29-34.

22. Otter JA, French GL. Nosocomial transmission of community-associated methicillin-resistant Staphylococcus aureus: An emerging threat. Lancet Infect Dis 2006;6:753-5.

23. Seybold U, Kourbatova EV, Johnson JG, Halvosa SJ, Wang YF, King MD, et al. Emergence of community-associated methicillin-resistant Staphylococcus aureus USA 300 genotype as a major cause of health care-associated blood stream infections. Clin Infect Dis 2006;42:647-66.

24. Patel M, Waites KB, Hoesley CJ, Stamm AM, Canupp KC, Moser SA. Emergence of USA300 MRSA in a tertiary medical centre: Implications for epidemiological studies. J Hosp Infect 2008;68:208-13.

25. Wagenvoort JH, De Brauwer EI, Sijstermans ML, Toenbreker HM. Risk of re-introduction of methicillin-resistant Staphylococcus aureus into the hospital by intrafamilial spread from and to healthcare workers. J Hosp Infect 2005;59:67-8

26. Deurenberg RH, Vink C, Kalenic S, Friedrich AW, Bruggeman CA, Stobberingh EE, et al. The molecular evolution of methicillin-resistant Staphylococcus aureus. Clin Microbiol Infect 2009;13:222-35.

27. Wang SH, Hines L, van Balen J, Mediavilla JR, Pan X,
Hoet $\mathrm{AE}$, et al. Molecular and clinical characteristics of hospital and community onset methicillin-resistant Staphylococcus aureus strains associated with bloodstream infections. J Clin Microbiol 2015;53:1599-608.

28. Reuter S, Ellington MJ, Cartwright EJ, Köser CU, Török ME, Gouliouris T, et al. Rapid bacterial whole-genome sequencing to enhance diagnostic and public health microbiology. JAMA Intern Med 2013;173:1397-404.

29. Harris SR, Cartwright EJ, Török ME, Holden MT, Brown NM, Ogilvy-Stuart AL, et al. Whole-genome sequencing for analysis of an outbreak of meticillin-resistant Staphylococcus aureus: A descriptive study. Lancet Infect Dis 2013;13:130-6.

30. De Faveri E, Rimoldi SG, Pagani C, Sala V. Interspecific epidemiology of MRSA in pig farming. Infect Dis Ther 2014;2:135.

31. European Committee on Antimicrobial Susceptibility Testing (EUCAST). Breakpoint Tables for Interpretation of MICs and Zone Diameters, Version 2.0; 2012. Available from: http://www.eucast.org/clinical_breakpoints. Accessed on 01-01-2012.

32. Enright MC, Day NP, Davies CE, Peacock SJ, Spratt BG. Multilocus sequence typing for characterization of methicillin-resistant and methicillin-susceptible clones of Staphylococcus aureus. J Clin Microbiol 2000;38:1008-15.

33. Gardner SN, Hall BG. When whole-genome alignments just won't work: KSNP v2 software for alignment-free SNP discovery and phylogenetics of hundreds of microbial genomes. PLoS One 2013;8:e81760.

34. Huson DH, Scornavacca C. Dendroscope 3: An interactive tool for rooted phylogenetic trees and networks. Syst Biol 2012;61:1061-7.

35. Chambers HF. The changing epidemiology of Staphylococcus aureus? Emerg Infect Dis 2001;7:178-82. 